\title{
Diffusion Tensor Imaging for Predicting the Outcome of Large-Vessel Ischemic Stroke Treated with Mechanical Thrombectomy: Is This the Prime Time?
}

D TI uses the physical principles of random displacement of water molecules, also known as Brownian motion, to measure the degree and directionality of water molecule motion in the human body. ${ }^{1}$ These measurements can be used to infer the microstructural integrity of the underlying tissue. In the brain healthy WM, cellular membranes, with some contribution from myelination and the packing of axons, skew the directionality of water diffusion, making it less uniform "i.e. anisotropic." ${ }^{2}$ Hence, lower fractional anisotropy (FA) measures have been found to reflect impairment of the WM microstructure. ${ }^{3}$ Since its introduction in the early 1990s, the rapid development of postprocessing techniques has led to widespread application of DTI in the various neurologic fields of study. For example, DTI has been successfully used to detect WM damage in traumatic brain injury, ${ }^{4}$ cerebral small-vessel disease, ${ }^{5}$ multiple sclerosis, and others. ${ }^{6}$ In the field of ischemic stroke, brain MR imaging, in particular DWI, has been instrumental in advancing the field with critical roles spanning from early detection of ischemic changes and patient selection for endovascular therapy to prognostication of functional outcome..$^{7-9}$ Indeed, larger DWI infarct volume has been associated with worse functional outcome after acute ischemic stroke. The introduction of mechanical thrombectomy in the management of patients with acute ischemic stroke, however, has significantly improved the functional outcome of patients with stroke with reduction in the associated infarct volume. ${ }^{8}$ In particular, after mechanical thrombectomy, infarction of the basal ganglia, secondary to the involvement of the perforator arteries that originate from the middle cerebral artery with sparing of the peripheral cortex, is now frequently encountered. Recent work has confirmed the role of the corticospinal tract (CST) injury by stroke in predicting motor recovery of the upper extremity. ${ }^{10}$ In this previous work, however, CST was mapped through the existing brain templates without direct measurement of its microstructural integrity. Hence, there is a need in the current thrombectomy era to better understand the outcomes of these patients and to guide selection in rehabilitation studies. DTI offers a sensitive tool that can address this dilemma by mapping the WM tracts, particularly the CST. Preliminary evidence of small studies suggests that DTI is suitable for this purpose. ${ }^{11}$ However, there remains a knowledge gap regarding the role of DTI in patients with stroke who are treated with mechanical thrombectomy.

In the article by Berndt et $\mathrm{al}^{12}$ the authors aim to answer this question by addressing the role of DTI in predicting the 3-month functional outcomes of patients with acute ischemic stroke caused by large-vessel occlusion in the anterior circulation who underwent mechanical thrombectomy. The study sample included 165 patients who had DTI sequences acquired within 7 days of stroke (median, 3 days). Probabilistic tractography was used to map the CST bilaterally, and atlas-based ROIs were used to characterize the CST in the posterior limb of the internal capsule (PLIC) and cerebral peduncle. Subsequently, the FA index was calculated for the PLIC and cerebral peduncle of the infarcted brain compared with healthy, nonaffected CST. The brain infarct was divided into the basal ganglia infarction (BGI) subgroup without relevant involvement of the cortical structures and the peripheral infarct subgroup in which the infarct involved the surrounding cortical areas. The latter's severity was further classified as mild, middle, or high, based on the peripheral territory involvement by the stroke that was originated from the Alberta Stroke Program Early CT Score (ASPECTS) of the DWI sequences. Good clinical functional outcome was defined by modified Rankin Scale score $\leq 2$ at 90 days.

The main results of this work confirm the lower FA index values of the CST on the stroke-affected side of the PLIC. Additionally, although there was no difference in the FA index of the CST between the BGI subgroup and the peripheral infarct subgroup, the latter subgroup showed a significantly higher FA index of the CST of the PLIC in those with mild infarction severity compared with middle or high infarction severity. Furthermore, in this subgroup, there were statistically significant negative correlations between the motor subindex of the National Institute of Health Stroke Scale (sum of arm and leg symptom value of the affected side) at the time of brain MR imaging and the FA index of the PLIC. From a long-term functional outcome standpoint, the FA index of the CST in the PLIC was significantly 
associated with good clinical outcomes in the study cohort. Although this effect was not seen in the BGI subgroup, a strong effect of the FA index on clinical outcome existed in the peripheral infarct subgroup. Finally, in mediation analysis, the effect of the infarction severity in the peripheral infarction subgroup on the 90-day functional outcome was present but reduced when controlling for the FA index of the CST, indicating that the FA index mediated the relationship between infarction severity and clinical outcome.

There are several notable accomplishments of this study. First, the sample size is relatively large, and the study population is homogeneous by including only patients with large-vessel occlusion stroke who were treated with mechanical thrombectomy. Second, the study ascertained the sensitivity of the FA index of the PLIC as an early measure of acute injury of CST in the setting of stroke. Third, the FA index of CST in the PLIC was also found to be a measure of the magnitude of motor deficit in the acute stroke phase in those with peripheral cortical stroke involvement. Finally, the FA index of the CST was associated with long-term functional outcome of acute ischemic stroke. Taken together, these results suggest that the FA index of the PLIC is a sensitive marker for acute CST injury after stroke, and it may represent a marker of long-term functional recovery.

This study, however, has several limitations. First, it did not include an accurate measure of the motor function acutely or at 3 months. Second, the degree of involvement of the peripheral cortical areas was measured through a categoric score rather than accurately segmenting the stroke area to assess the degree of anatomic overlap with the CST. Third, the study did not include a repeat DTI scan at 3 months to assess whether the improvement of functional outcome coincided with improvement on the FA index of the CST. Finally, the underlying causes of impairment of the microstructural integrity of the CST cannot be ascertained; it is unknown whether it is caused by tissue necrosis from stroke, edema, or Wallerian degeneration. Knowing the cause is necessary to understand the reversibility of the CST injury.

In summary, although the current study is a step forward in incorporating DTI into the clinical care of patients with stroke, several important questions first need to be resolved in large prospective studies to achieve this goal. These include, among many others, determining the relevant WM tracts for recovery, defining the critical FA thresholds of the necessary tracts for motor and functional improvement, characterizing types and location of injury, and selecting patients who will benefit from rehabilitation. Addressing these important questions will bring DTI closer for application in the daily care of patients with ischemic stroke. When combined with other genetic and serologic biomarkers of recovery, this will enable physicians to accurately predict the functional outcome of acute ischemic stroke through personalized medicine. ${ }^{13}$ Advanced imaging acquisition and analytics, including DTI, are at the forefront of these efforts, and they are ready to be tested in large multicenter clinical trials.

\section{Conflict of Interest}

Author reports no conflicts of interests in relationship to this work.

\section{REFERENCES}

1. Yang E, Nucifora PG, Melhem ER. Diffusion MR imaging: basic principles. Neuroimaging Clin N Am 2011;21:1-25, vii CrossRef Medline

2. O'Donnell LJ, Westin CF. An introduction to diffusion tensor image analysis. Neurosurg Clin N Am 2011;22:185-96, viii CrossRef Medline

3. Tournier JD, Mori S, Leemans A. Diffusion tensor imaging and beyond. Magn Reson Med 2011;65:1532-56 CrossRef Medline

4. Asken BM, DeKosky ST, Clugston JR, et al. Diffusion tensor imaging (DTI) findings in adult civilian, military, and sport-related mild traumatic brain injury (mTBI): a systematic critical review. Brain Imaging Behav 2018;12:585-612 CrossRef Medline

5. Hannawi Y, Yanek LR, Kral BG, et al. Hypertension is associated with white matter disruption in apparently healthy middle-aged individuals. AJNR Am J Neuroradiol 2018;39:2243-48 CrossRef Medline

6. Tae WS, Ham BJ, Pyun SB, et al. Current clinical applications of diffusion-tensor imaging in neurological disorders. J Clin Neurol 2018;14:129-40 CrossRef Medline

7. Bang OY, Li W. Applications of diffusion-weighted imaging in diagnosis, evaluation, and treatment of acute ischemic stroke. Precis Future Med 2019;3:69-76 CrossRef

8. Albers GW, Marks MP, Kemp S, et al. Thrombectomy for stroke at 6 to 16 hours with selection by perfusion imaging. $N$ Engl J Med 2018;378:708-18 CrossRef Medline

9. Thomalla G, Simonsen CZ, Boutitie F, et al. MRI-guided thrombolysis for stroke with unknown time of onset. N Engl J Med 2018;379:61122 CrossRef Medline

10. Lin DJ, Cloutier AM, Erler KS, et al. Corticospinal tract injury estimated from acute stroke imaging predicts upper extremity motor recovery after stroke. Stroke 2019;50:3569-77 CrossRef Medline

11. Puig J, Blasco G, Schlaug G, et al. Diffusion tensor imaging as a prognostic biomarker for motor recovery and rehabilitation after stroke. Neuroradiology 2017;59:343-51 CrossRef Medline

12. Berndt MT, Purner D, Maegerlein C, et al. Basal ganglia versus peripheral infarcts: predictive value of early fiber alterations. AJNR Am J Neuroradiol 2020;42:264-70 CrossRef Medline

13. Hinman JD, Rost NS, Leung TW, et al. Principles of precision medicine in stroke. J Neurol Neurosurg Psychiatry 2017;88:54-61 CrossRef Medline

(1) Y. Hannawi Division of Cerebrovascular Diseases and Neurocritical Care Department of Neurology, The Ohio State University

http://dx.doi.org/10.3174/ajnr.A7026

Columbus, Ohio 